\title{
Periodic Autoregressive Forecasting of Global Solar Irradiation Without Knowledge-based Model Implementation
}

\author{
Cyril Voyant $^{a, c, *}$ Jan G. De Gooijer ${ }^{b}$, Gilles Notton ${ }^{c}$ \\ ${ }^{a}$ University of Corsica, CNRS UMPR SPE 6134, 20250 Corse, France \\ ${ }^{b}$ University of Amsterdam, 1018 WB Amsterdam, the Netherlands \\ ${ }^{c}$ Hospital of Castelluccio, Radiotherapy Unit, BP 85, 20177 Ajaccio, France
}

\begin{abstract}
Reliable forecasting methods increase the integration level of stochastic production and reduce cost of intermittence of photovoltaic production. This paper proposes a solar forecasting model for short time horizons, i.e. one to six hours ahead. In this time-range, machine learning methods have proven their efficiency. But their application requires that the solar irradiation time series is stationary which can be realized by calculating the clear sky global horizontal solar irradiance index (CSI), depending on certain meteorological parameters. This step is delicate and often generates additional uncertainty if conditions underlying the calculation of the CSI are not well-defined and/or unknown. As a novel alternative, we introduce a so-called periodic autoregressive (PAR) model. We discuss the computation of post-sample point forecasts and forecast intervals. We show the forecasting accuracy of the model via a real data set, i.e., the global horizontal solar irradiation (GHI) measured at two meteorological stations located at Corsica Island, France. In particular, and as opposed to methods based on CSI, a PAR model helps to improve forecast accuracy, especially for short forecast horizons. In all the cases, PAR is more appropriate than persistence, and smart persistence. Moreover, smart persistence based on the typical meteorological year gives more reliable results than when based on CSI.
\end{abstract}

Keywords: Autoregression; Clear sky irradiance; Forecast intervals; Periodic

\footnotetext{
${ }^{*}$ Corresponding author. Tel.: +33 4952936 66; fax: +33495293797.

E-mail address: cyrilvoyant@gmail.com
} 


\section{$1 \quad 1$ Introduction}

2 Solar energy, mainly photovoltaic, is an energy resource which plays an increasingly important 3 role in the electrical energy production due to its abundance, cleanness and cost effectiveness

4 characteristics with limited environmental consequences. On the other hand, solar power has a 5 fluctuating generation profile because of its inherent cyclic and time varying nature, leading to 6 limitations on stability and trustworthiness of solar power grid systems (Shamshirband et al., 7 2015). To reduce the inconvenience of this stochastic and intermittent nature, and to improve the 8 inclusion of solar power plants, an efficient forecasting method of solar radiation is paramount.

9 Moreover, this intermittent character gives rise to additional production costs compared with 10 conventional production, from 1 to $8 € / \mathrm{MWh}$ with an average value around $6 € / \mathrm{MWh}$; Notton et 1 al. (2018). Thus, a reliable production forecasting method decreases the average annual operating 2 costs. In addition, it reduces the reserve shortfalls and it reduces curtailments. Several methods 3 are available for forecasting depending on the time horizon and time resolution (Notton and 14 Voyant, 2018).

This paper concerns forecasting at short time horizons, i.e., one to six hours ahead with a one hour time step. In this time-range, machine learning methods have proven their accuracy. But their application requires that a solar irradiation time series is stationary which can be realized by calculating conditions for clear sky (CS) solar irradiation, depending on certain meteorological parameters (Diagne et al., 2013; Lauret et al., 2015). The use of a CS solar radiation model, however, induces an important source of error because this type of model depends on meteorological parameters which vary month by month or during a day. To avoid this difficulty, the purpose of the paper is to present a forecasting model which does not require a CS model, and which can be easily implemented in practice.

The remainder of the paper is organized as follows. In Section 2, we introduce the concept of periodically correlated processes and we provide arguments why global horizontal solar irradiation measurements are periodic seasonal time series. In Section 3, we discuss problems induced by a CS model. Section 4 provides details about the data under study. The periodic autoregressive (PAR) model is introduced in Section 5, and expressions for point forecasts, forecast intervals, and forecast evaluation measures are given. Section 6 provides some information about alternative forecasting models. Section 7 shows PAR identification and PAR forecasting results. It includes results of a comparative forecasting experiment. Lastly, Section 8 offers some concluding remarks. 


\section{Periodic Phenomena}

Given a stationary time series $\left\{Y_{t} ; t \in \mathbb{Z}\right\}$ whose second order moments exist, we define its mean function by $\mu_{t}=\mathbb{E}\left(Y_{t}\right)$ and its autocovariance function by $\gamma_{s, t}=\operatorname{Cov}\left(Y_{s}, Y_{t}\right)$. The process is said to be periodically correlated (PC) with period $H$, or periodic stationary (Gladyšhev, 1961), if the following two conditions

$$
\mu_{t}=\mu_{t+h} \text { for all } t \text {, and }
$$

$$
\gamma_{s, t}=\gamma_{s+h, t+h} \text { for every } s, t \text { in the index set }
$$

are true for $h=H$ but for no smaller value of $h$. Note that the case where $\mu_{t}$ and $\gamma_{s, t}$ do not depend on $t$ reduces to the ordinary covariance stationary time series. Throughout the rest of the paper, we assume without loss of generality that $\mu_{t}=0$. The periodic autocorrelation function at lag $s=1,2, \ldots$ and time $t$ is defined by $\rho_{s, t}=\gamma_{s, t} / \gamma_{0, t}$ which is the theoretical counterpart of the autocorrelation function at lag $s$ of a stationary time series.

Global horizontal solar irradiation (GHI, in $\mathrm{Wh} / \mathrm{m}^{2}$ ) measurements can be viewed as periodic seasonal time series. In general, a seasonal pattern appears when a time series is influenced by seasonal factors, e.g., the month of the year, the day of the week, or the hour of the day; Hokoi et al. (1990). As can be seen from (1) the seasonality is always of a fixed and known period, and hence, the time series is called periodic. In general, the average length of cycles is longer than the length of a seasonal pattern, and the magnitude of cycles tends to be more variable than the magnitude of seasonal patterns; Franses and Paap (1994). From these observations, we deduce the following two properties.

1) The observed time series $\operatorname{GHI}(t)(t=1, \ldots, N)$ can be considered as a periodic time series with two fixed seasonal periods $H$ and $D$. In this study $H=24$ hours $(h)$ and $D=365$ days $(d)$. For simplicity, we assume that $N /(H \times D)=Y$ is an integer representing the number of available years. The decomposition of $\operatorname{GHI}(t)$ highlights three new time series: two are strongly seasonal, and one time series is related to the noise, or irregular component. That is

$$
\{\mathrm{GHI}(t), t \in \mathbb{Z}\}=\left\{f\left(\mathrm{~S}_{24 h}(t), \mathrm{S}_{365 d}(t), \varepsilon(t)\right), t \in \mathbb{Z}\right\}
$$

2) The function $f(\cdot)$ defines the type of decomposition: additive, multiplicative or hybrid. Usually the multiplicative mode is preferred, and the term $\mathrm{S}_{24 h} \times \mathrm{S}_{365 d}$ at time $t$ is a proxy of the so-called CS global irradiation, i.e., $\mathrm{CS}(t)=\left\{S_{24 h}(t) \times S_{365 d}(t), t \in \mathbb{Z}\right\}$. 
The ratio $\operatorname{GHI}(t) / \mathrm{CS}(t)$ defines the clear sky index $\operatorname{CSI}(t) \in[0,1]$.

Observe from property 1) that a solar irradiation time series contains only seasonal patterns. These components can be deleted by seasonal adjustment using a ratio to trend (detrending), divided by an estimate of a CS $(t)$ series (Grantham et al., 2016, 2018) or, if estimation is difficult, divided by a moving average of the series (Voyant et al., 2011). Alternatively, one can adopt a classical seasonal autoregressive integrated moving average (SARIMA) model. Implicit in such models is the assumption of homogeneity or time invariance, i.e. the seasonally differenced series is sure to become stationary. However, many seasonal time series cannot be filtered, standardized or differenced to achieve second-order stationarity because the series exhibits a strong seasonal behavior such that the entire autocorrelation structure of the series depends on the season, hence the homogeneity assumption fails (Tiao and Grupe, 1980). In fact, the majority of GHI time series satisfy the property of periodic stationarity (Ula and Smadi, 1997), stating that their sample mean and sample autocorrelation function are periodic with respect to time. A more realistic family of models characterizing those kind of seasonal time series is the PAR model.

The method of moments based on the well-known Yule-Walker equations and the least squares method in the univariate case are both efficient ways to estimate PAR models. In particular, when the seasonal data and the model for each season are used rather than the annual data, significant gains in parameter efficiency can be achieved. However, the number of estimated parameters is likely to increase with the choice of the season. Thus, in our study it will be easier to consider only the $H=24$ hours period, giving rise to a parsimonious PAR model with only 24 components rather than estimating a model with $D=365$ components. Moreover, it is often useful to put linear constraints on the parameters for a given season. Another important advantage is that PAR models can be studied for each season separately. It justifies the use of AIC and BIC information criteria and sample periodic (partial) autocorrelations to identify the optimal model order.

In the next section, we will focus on two approaches to take seasonality into account. The first approach uses a white box model (WM) based on the knowledge model which we couple with the stochastic modeling of CSI $(t)$. This approach is often called grey box modeling, or in short-hand notation GM. The second approach uses the previously measured data and any knowledge-based model, and we call it a black box model or BM. Observe that a GM $(=\mathrm{WM}+\mathrm{BM})$ is often more interesting to analyze than a BM since it encompasses a semi-physical model. But adopting the GM can add an additional uncertainty if the parameters of the model are not well-defined, and thus decreasing the reliability of the GM. 


\section{Clear Sky (CS) Model}

For a temporal forecast horizon up to and including six hours ahead, a CS solar irradiation model is often used to make the time series $\operatorname{GHI}(t)$ stationary, before calculating the $\mathrm{CS}(t)$ index (Lauret et al., 2015; Voyant et al., 2015). The chosen CS model in this study is the Solis model (Mueller et al., 2004; Ineichen, 2008). The CS global horizontal irradiance reaching the ground is defined by

$$
\mathrm{CS}(t)=I_{0}(t) \exp \left(\frac{-\tau}{\sin ^{g}(\eta(t))}\right) \sin (\eta(t)) .
$$

Here $I_{0}$ is the extraterrestrial radiation (depends on the day of the year), $\eta$ is the solar elevation angle (depends on the hour of the day), $\tau$ is the global total atmospheric optical depth (depends on the day of the year and the hour of the day), and $g$ is a fitting parameter. In order to be welldefined, the CS model requires meteorological parameters (Gelaro et al., 2017) to characterize the state of the sky such as, for instance, the aerosol optical depth (AOD) and the water vapor column defining the total AOD. These parameters are difficult to obtain. Moreover, they fluctuate in a large range from one year to another and during a day from one hour to the next. Thus, the average value of these parameters do not accurately reflect the CS condition at a given time $t$. Indeed, Voyant et al. (2015, Figure 5) showed the impact of AOD values on the forecast accuracy, as measured by the normalized mean absolute forecasting error (nMAE) for Ajaccio. Specifically, these authors obtain an nMAE value of $11 \%$ in the optimized parameter case, and $18 \%$ with very ill-optimized parameters, so an increase of 7 percentage points.

Moreover, obtaining accurate $\operatorname{CSI}(t)$ series at sunset and sunrise is difficult due to a possible surrounding masking effect such as mountains, buildings, or vegetation. It may also be due to unreliable measurements of solar irradiation at low solar height (instrumental errors due to the cosine response). For these reasons, a pre-processing operation is applied: solar radiation data for which the solar elevation is lower than a threshold of $5^{\circ}$ or $10^{\circ}$ are removed from the analysis. However, the solar production during these sunset and sunrise periods are often non negligible and their forecasts cannot be avoided. For forecasting tilted solar irradiation, a CS model uses a constant albedo which, in practice, varies seasonally and sometimes during the day (modifications of the land cover, Notton et al., 2006). For our experimental site, the influence of the sea on the reflected and diffused solar radiation differs in the morning and in the evening; Ineichen et al. (1990). Finally, some time lags can occur between the measured and the modeled CS irradiances due to time synchronization or the use of various time scales. 


\section{Data}

Time series of $\mathrm{GHI}(t)$ observations measured at Ajaccio $\left(41^{\circ} 55^{\prime} \mathrm{N}, 8^{\circ} 44^{\prime} \mathrm{E}, 4 \mathrm{~m}\right.$ asl $)$ and Bastia $\left(42^{\circ} 42^{\prime} \mathrm{N}, 9^{\circ} 27^{\prime} \mathrm{E}, 10 \mathrm{~m}\right.$ asl $)$ meteorological stations, Corsica island, France. Both stations are located near the Mediterranean Sea and nearby mountains (more than $1000 \mathrm{~m}$ altitude at 40 $\mathrm{km}$ from the two sites). This specific geographical configuration makes nebulosity or "cloud-likeness" difficult to forecast. The climate of Corsica is characterized by hot summers with abundant sunshine and mild, dry and clear winters. For Ajaccio, hourly global horizontal solar radiation data are available for the time period 1998-2008 (11 years) and for Bastia the data covers the period 2003-2008 (6 years).

A standard cleaning approach is applied to identify and remove bad data. Often mistakes appear in time series of solar data due to problems with the acquisition system; an automatic quality check used in the frame of GEOSS project (Group on Earth Observation System of System) has been applied to the data. The quality of the data (Korany et al., 2016) and the procedure applied to flag suspicious or erroneous measurements is described in detail by David et al. (2016). Both stations are equipped with pyranometers (CM 11 Kipp \& Zonen) and standard meteorological sensors (pressure, nebulosity, etc.). A filtering approach is first applied before computing the various time series models. GHI values linked to a solar elevation angle less than $10^{\circ}$ are excluded from the analysis. In that case, forecasting GHI values will be very difficult due to inaccurate CSI values obtained between periods of sunset and sunrise.

The comparison between machine learning models and PAR models is done during the last year of the available data set covering $365 \times 24=8,760$ observations. For Ajaccio the first 10 years $(87,600$ observations) are used as a training set of the machine learning models, while there are 5 years of daily observations in the training set for Bastia. To reduce computation time, estimation is done only once using the training/estimation period.

\section{Periodic Autoregression}

\section{$5.1 \quad$ Model}

Machine learning models are usually adopted to predict GHI (Voyant et al., 2017). They are often based on the assumption that the data generation process does not change over time, so assuming that the time series under study is stationary. As noted above, the process to make a time series stationary can be complex and, more importantly, can generate uncertainties. In 
contrast, a time series fitted by a PAR model does not need the stationarity assumption. In fact, by definition (1) the time series process is said to be periodically stationary. Moreover, PAR models avoid CS modeling.

Let $\{y \in \mathbb{Z} \mid 1 \leq y \leq Y\}$ denote the set of years with $Y$ the number of within-in-sample years. Similarly, let $\{d \in \mathbb{Z} \mid 1 \leq d \leq D\}$ the set of days at year $y$, and $\{h \in \mathbb{Z} \mid 1 \leq h \leq H\}$ the set of hours at day $d$. So, the time index parameter $t$ may be written as $t \equiv t(y, d, h)=$ $H \times D(y-1)+H(d-1)+h$. Then, a PAR stochastic process model of period $h(h=1, \ldots, H)$ and order $p(h)$, for the $\operatorname{GHI}(t)$ process is defined by

$$
\operatorname{GHI}(t)=\sum_{k=1}^{p(h)} \phi_{k}(h) \mathrm{GHI}(t-k)+\varepsilon(t),
$$

where $\phi_{k}(h)$ are the autoregressive (AR) coefficients at hour $h$, and $\varepsilon(t)$ is a periodic white noise process with $\mathbb{E}(\varepsilon(t))=0$ and $\operatorname{Var}(\epsilon(t))=\sigma^{2}(h)$, independent of $\operatorname{GHI}(t)$. When $h=d=1$, model (3) reduces to a "classical" AR model.

Let $\operatorname{GHI}(y, d, h+H)=\operatorname{GHI}(y, d+1, h), \operatorname{GHI}(y, d+D, h)=\mathrm{GHI}(y+1, d, h), \phi_{k}(h+H)=$ $\phi_{k}(h)$, and $p(h+H)=p(h)$. Then, with $\operatorname{GHI}(t) \equiv \operatorname{GHI}(y, d, h)$ and $\varepsilon(t) \equiv \varepsilon(y, d, h)$, Eq. (3) can be rewritten as

$$
\operatorname{GHI}(y, d, h)=\sum_{k=1}^{p(h)} \phi_{k}(h) \operatorname{GHI}(y, d, h-k)+\varepsilon(y, d, h) .
$$

A convenient way to represent Eq. (4) is by using vector notation,. That is, for each hour $h$, the daily and yearly observations are stacked in the $(D \times Y)$-dimensional column vector $\mathbf{Y}(h)=(\operatorname{GHI}(1,1, h), \operatorname{GHI}(1,2, h), \ldots, \operatorname{GHI}(Y, D, h))^{\prime}$. Model $(4)$ can then be written as

$$
\mathbf{Y}(h)=\mathbf{X}(h) \mathbf{\Phi}(h)+\varepsilon(h),
$$

with

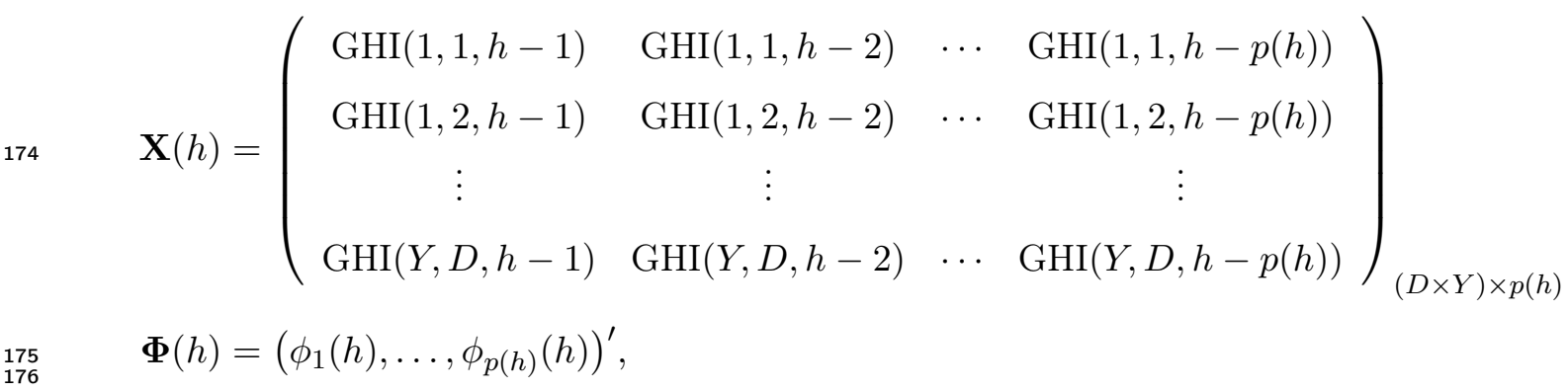

where $\varepsilon(h)$ is a $(D \times Y)$-dimensional column vector containing the stacked daily and yearly white noise errors corresponding to $\mathbf{Y}(h)$. The parameter vector $\mathbf{\Phi}(h)$ can be estimated by least 
squares (LS). In particular, an estimator of $\mathbf{\Phi}(h)$ is given by $\widehat{\mathbf{\Phi}}(h)=\left(\widehat{\phi}_{1}(h), \ldots, \widehat{\phi}_{p(h)}(h)\right)^{\prime}=$ $\left[\left\{\mathbf{X}^{\prime}(h) \mathbf{X}(h)\right\}^{-1} \mathbf{X}^{\prime}(h)\right] \mathbf{Y}(h)$ and the corresponding residuals are defined by $\widehat{\varepsilon}(h)=\mathbf{Y}(h)-$ $\mathbf{X}(h) \widehat{\mathbf{\Phi}}(h)$.

Model (4) can be enlarged by considering yearly varying trends and constants. In the numerical study, we estimate a PAR model with a constant related to a synthetic sequence of hourly global horizontal irradiation denoted in this study by an approximation of the "typical meteorological year" (TMY, Grantham et al. (2018)) or climatology. That is $\operatorname{TMY}(d, h)=$ $Y^{-1} \sum_{y=1}^{Y} \operatorname{GHI}(y, d, h)$. In that case a PAR model can be written as

$$
\operatorname{GHI}(y, d, h)-\operatorname{TMY}(d, h)=\sum_{k=1}^{p(h)} \phi_{k}(h)(\operatorname{GHI}(y, d, h-k)-\operatorname{TMY}(d, h-k))+\varepsilon(y, d, h) .
$$

\section{$5.2 \quad$ Forecasting}

Assuming that Eq. (6) is known in the sense that all the parameters are known, the optimal one-step ahead forecast is given by

$\widehat{\operatorname{GHI}}(y, d, h+1)=\sum_{k=1}^{p(h)} \phi_{k}(h)(\operatorname{GHI}(y, d, h-k+1)-\operatorname{TMY}(y, d, h-k+1))+\operatorname{TMY}(y, d, h+1)$.

The corresponding forecast error is $\operatorname{GHI}(y, d, h+1)-\widehat{\mathrm{GHI}}(y, d, h+1)=\varepsilon(y, d, h+1)$, and the forecast error variance is $\sigma^{2}(h)$. In practice the coefficients $\phi_{k}(h)$ are replaced by estimates $\widehat{\phi}_{k}(h)$.

The optimal $\ell$-step ahead forecast $(\ell>1)$ is given by

$$
\begin{aligned}
\widehat{\operatorname{GHI}}(y, d, h+\ell)= & \sum_{k=1}^{p(h+\ell-1)} \phi_{k}(h+\ell-1)(\widehat{\operatorname{GHI}}(y, d, h-k+\ell-1)-\operatorname{TMY}(d, h-k+\ell-1)) \\
& +\operatorname{TMY}(y, d, h+\ell) .
\end{aligned}
$$

The corresponding $\ell$-step ahead forecast error is given by

$$
\operatorname{GHI}(y, d, h+\ell)-\widehat{\operatorname{GHI}}(y, d, h+\ell)=\varepsilon(y, d, h+\ell)+\sum_{i=1}^{\ell-1}\left(\varepsilon(y, d, h+i) \prod_{j=i}^{\ell-1} \phi_{h+j}^{*}\right),
$$

where $\phi_{h}^{*}=\sum_{k=1}^{p(h)} \phi_{k}(h)$. The forecast error variance is given as

$$
\sigma^{2}(h, \ell)=\sigma^{2}(h)\left\{1+\sum_{i=1}^{\ell-1}\left(\prod_{j=i}^{\ell-1}\left(\phi_{h+j}^{*}\right)^{2}\right)\right\}
$$




\subsection{Forecast intervals (FIs)}

On the assumption that the forecast errors are normally distributed, a standard Box-Jenkins FI for an $\ell$-step ahead forecast of GHI is of the form $\widehat{\mathrm{GHI}}(y, d, h+\ell) \pm z_{\alpha / 2} \sigma(h, \ell)$ where $z_{\alpha / 2}$ denotes the $(1-\alpha / 2)$ th percentile of the standard normal distribution. However, a preliminary analysis of both time series indicated non-normality ( $p$-values $<0.001)$ of the distribution of $\widehat{\operatorname{GHI}}(y, d, h+\ell)$ with significant values of the sample skewness and kurtosis. In this case, bootstrapping FIs (BFIs) is a possible alternative.

Now, assuming a PAR model is correctly specified, the residuals from a fitted PAR model are asymptotically uncorrelated (McLeod, 1994). This result implies that bootstrapping can be carried for each hour $h$ separately. BFIs for ARs have received quite some attention; see, e.g., Pan and Politis (2016) for a recent review. In this paper, we use their Algorithm 3.5 (backward bootstrap with fitted residuals). Monte Carlo simulations performed by these authors show that this algorithm performs well in terms of coverage. For a fixed $h$, the resulting $\ell$ step ahead coverage probability $(1-\alpha)$ will be denoted by

$$
\mathrm{BFI}_{\alpha}(\ell)=\left[{\widehat{\mathrm{GHI}_{B}}}^{(\alpha / 2)}(t+\ell),{\widehat{\mathrm{GHI}_{B}}}^{(1-\alpha / 2)}(t+\ell)\right],
$$

where $\widehat{\mathrm{GHI}}_{B}^{(\alpha / 2)}(t+\ell)$ and $\left.\widehat{\mathrm{GHI}}_{B}^{(1-\alpha / 2)}(t+\ell)\right]$ are, respectively, the $(\alpha / 2)$ th and $(1-\alpha / 2)$ th percentiles of the empirical bootstrapped distribution function of $\mathrm{GHI}(t+\ell)$ based on $B$ bootstrap replicates. We set $B=1,000$ in our computation of FIs (Section 7.3).

\subsection{Forecast Evaluation}

We use three error measures to compare the out-of-sample forecasting performance of the models. The first criterion is the normalized root mean square error (nRMSE, unitless); it is a commonly used error metric for evaluating point forecasts of GHI; see, e.g., Lauret et al. (2015). The measure is defined as $\operatorname{nRMSE}(\ell)=\left[\sum_{t=1}^{N(\ell)}(\widehat{\mathrm{GHI}}(t)-\operatorname{GHI}(t))^{2}\right]^{1 / 2} / \sum_{t=1}^{N} \operatorname{GHI}(t)$, where $N(\ell)$ is the number of out-of-sample forecasts depending on forecast horizon $\ell=1, \ldots, 6$. The second measure is the normalized mean interval length (nMIL; unitless). For a coverage probability $(1-\alpha)$ and given set of $\ell$-step ahead forecasts $\{\widehat{\mathrm{GHI}}(t+\ell)\}$, this measure is defined as the difference between the upper $\widehat{\mathrm{GHI}}_{\mathrm{U}}^{(1-\alpha)}(t+\ell)$ and lower $\widehat{\mathrm{GHI}}_{\mathrm{L}}^{(1-\alpha)}(t+\ell)$ limits. The resulting statistic is given by $\operatorname{nMIL}^{(1-\alpha)}(\ell)=\sum_{t=1}^{N(\ell)}\left(\widehat{\mathrm{GHI}}_{\mathrm{U}}^{(1-\alpha)}(t)-\widehat{\mathrm{GHI}}_{\mathrm{L}}^{(1-\alpha)}(t)\right) / \sum_{t=1}^{N(\ell)} \mathrm{GHI}(t)$. As a third evaluation measure, we consider the $\ell$-step ahead prediction interval coverage $\operatorname{PICP}^{(1-\alpha)}(\ell)$. This measure is defined by the probability that the target value of an input pattern lies between the forecast limits. That is, $\operatorname{PICP}^{(1-\alpha)}(\ell)=100 \times(1 / N(\ell)) \#(j)$ with $\#(j)$ the number of $j$ 's 
Table 1: Summary of forecasting models. Short-hand notation: $\mathrm{SP}=$ smart persistence, AR $=$ autoregressive, $\mathrm{MLP}=$ multilayer perceptron, $\mathrm{PAR}=$ periodic $\mathrm{AR}, \mathrm{TMY}=$ typical meteorological year, $\mathrm{CS}=$ clear sky (Solis) model.

\begin{tabular}{|c|c|c|}
\hline $\begin{array}{l}\text { Knowledge-based } \\
\text { model }\end{array}$ & $\begin{array}{l}\text { Predictor } \\
\text { type }\end{array}$ & $\begin{array}{l}\text { Form of the } \ell \text {-step ahead forecast } \\
\widehat{\operatorname{GHI}}(y, d, h+\ell)\end{array}$ \\
\hline \multirow[t]{3}{*}{ With } & SP1 & $\operatorname{GHI}(y, d, h) \frac{\operatorname{CS}(d, h+\ell)}{\operatorname{CS}(d, h)}$ \\
\hline & $\operatorname{AR}(p)$ & $\sum_{i=1}^{p} \frac{\phi_{i} \operatorname{GHI}(y, d, h+\ell-i)}{\operatorname{CS}(d, h+\ell-i)} \operatorname{CS}(d, h+\ell)$ \\
\hline & $\mathrm{MLP}^{*}$ & $\sum_{j=1}^{m} w_{j} f\left(\sum_{i=0}^{p} \frac{w_{j i} \operatorname{GHI}(y, d, h-i)}{\operatorname{CS}(d, h-i)}\right) \operatorname{CS}(d, h+\ell)$ \\
\hline \multirow[t]{2}{*}{ Without } & $\mathrm{SP} 2$ & $\operatorname{GHI}(y, d, h)-\operatorname{TMY}(d, h)+\operatorname{TMY}(d, h+\ell)$ \\
\hline & $\operatorname{PAR}(p(h))$ & Equation (7) \\
\hline
\end{tabular}

* An AR-NN model of order $p$ with $m$ hidden neurons and a single linear output.

The initial weights are denoted by $w_{j}$ and $w_{j i}$.

237 lying in the interval $\left[\widehat{\mathrm{GHI}}_{\mathrm{L}}^{(1-\alpha)}(t), \widehat{\mathrm{GHI}}_{\mathrm{U}}^{(1-\alpha)}(t)\right]$. Several test statistics can be based on PICP;

238 see, e.g., De Gooijer (2017, Chapter 10).

\section{Some Alternative Models}

It is interesting to compare the proposed PAR forecasting model with existing naïve and reference models. Naïve models will be denoted by SP which stands for smart persistence. They are simple models related to the knowledge-based model CS (SP1) and to TMY (SP2) as described in Table 1. Detailed information about CS and SP1 can be found in Lauret et al. (2015), Voyant et al. (2015), Mueller et al. (2004), and Ineichen (2008) and the references therein. The CS model under study is the so-called Solis model which is known to give good results; see, e.g., Lauret et al. (2015), Ruiz-Arias et al. (2017), and Voyant et al. (2015). Two reference models are an AR model and a multilayer perceptron (MLP) belonging to the class of artificial neural network models. The main equations are given in Table 1; see, e.g., Voyant et al. (2011), Voyant et al. (2014), and Voyant et al. (2017). 


\section{$7 \quad$ Empirical Results}

\subsection{Exploratory Analysis}

The presence of periodic correlation in the series $\mathrm{GHI}(t)$ can be detected by the sample periodic autocorrelation function at time lag $s$ and period $h$, i.e. $r_{s, h}=c_{s, h} /\left(c_{0, h} c_{0, h-s}\right)^{1 / 2}$ where $c_{s, h}=$ $Y^{-1} \sum_{y}(\operatorname{GHI}(y, d, h)-\operatorname{TMY}(d, h))(\operatorname{GHI}(y-s, d, h)-\operatorname{TMY}(d, h))(y=1, \ldots, Y ; h=1, \ldots, H)$. This statistic can also be used to test the null hypothesis: $\rho_{s, h} \equiv \rho_{s},(s=1,2, \ldots)$. Using the function peracf in the R-perARMA package, we reject the null hypothesis for all values $s=$ $1, \ldots, 30$ ( $p$-values $<0.001)$. This indicates that both GHI $(t)$ series are properly PC and is not just an amplitude modulated stationary sequence. In addition, we reject the null hypothesis $\rho_{s, h}=0(p$-values $<0.001)$. So the series are not PC white noise.

A suitable PAR model can be selected by examining plots of the sample periodic partial autocorrelation (perPACF), say $\widehat{\rho}_{\cdot, s, h}$, or by using an information criterion such as AIC or BIC. Sakai (1982) showed that if the correct order is $p(h)$ for period $h$, the estimate of the asymptotic standard deviation of $\widehat{\rho}_{\cdot, s, h}$ equals $1 / \sqrt{n}, s>p(h)$. The order $p(h)$ can be identified by finding the lowest lag for which the sample perPACF cuts off. BIC may be factored to obtain a separate criterion for each period. Thus

$$
\operatorname{BIC}(p(1), \ldots, p(H))=\sum_{h=1}^{H} \operatorname{BIC}(p(h))
$$

with

$$
\operatorname{BIC}(p(h))=\log \left(\widehat{\sigma}_{h}^{2}(p(h))\right)+\frac{\log (n)}{n} p(h),
$$

where $\widehat{\sigma}_{h}^{2}(p(h))$ corresponds to the LS estimator of the residual variance, and $n=3,650(1,825)$ in the case of Ajaccio (Bastia). Thus, the total minimization of Eq. (10) can be established by minimizing each $\operatorname{BIC}(p(h))$ about $p(h)$. Replacing $\log (n)$ in Eq. (11) by 2 gives AIC. It should be pointed out that for identifying subsets of PAR models with AIC or BIC a local search algorithm (e.g., a genetic algorithm, Ursu and Turkman, 2012) is recommended to avoid lengthy computations.

Table 2 summarizes the significance of the sample perPACF values for Ajaccio at a $5 \%$ nominal significance level, in terms of two indicator symbols and for $h=4, \ldots, 20$. Since global solar radiation is zero during the night, depending on the season, the sunrise and the fluctuation of sunshine, sample perPACF results for $h=1,2,3$ and $h=21, \ldots, 24$ are not included. Observe that for almost all values of $h$, significant values of $\widehat{\rho}_{\cdot, s, h}$ occur at lags $s=1,2,3$. For $h=$ 
Table 2: Indicator pattern of statistically significant values of the sample perPACF for Ajaccio; "+" indicates a value $>1.96 n^{-1 / 2}$ and "-_" a value $<-1.96 n^{-1 / 2}$ with $n=3,650$.

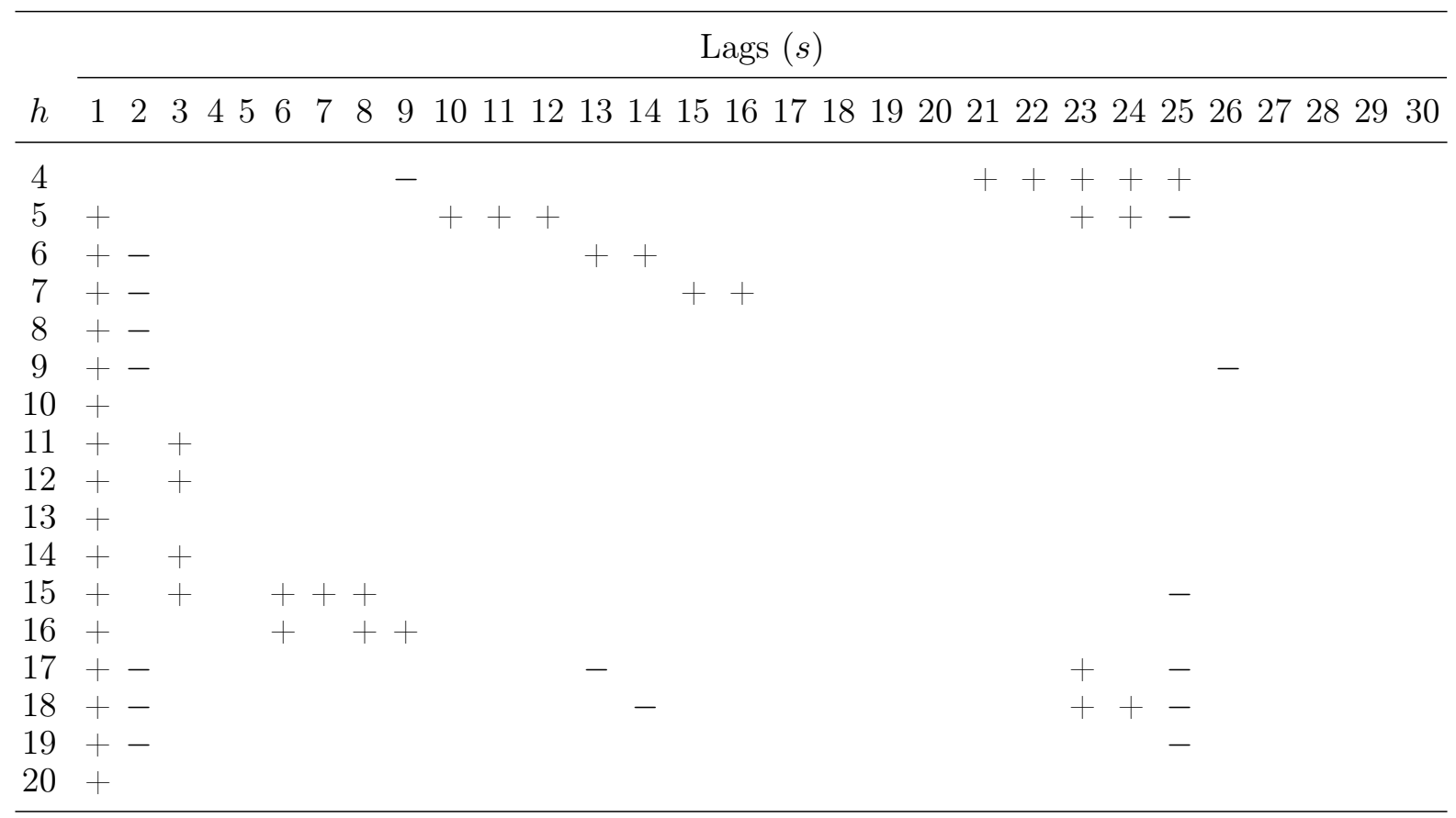

$4, \ldots, 20$, AIC selects a PAR model of order $(24,25,25,26,18,2,3,1,3,7,4,7,8,12,26,25,26)$ at forecast horizon $\ell=1$ while $\operatorname{BIC}$ suggests a $\operatorname{PAR}(24,25,25,14,2,2,2,1,3,4,1,3,8,9,26,25,2)$ model. These results are broadly in agreement with the orders selected by perPACF for the GHI series of Ajaccio. Note, BIC prefers low order PAR models. Similar results are observed for the GHI series of Bastia at $\ell=1$. Forecast results will be based on the PAR model orders determined by AIC and BIC.

\section{$7.2 \quad$ Forecasting}

The nRMSE forecasting results are summarized in Table 3, where PAR-AIC (PAR-BIC) denote the PAR models selected by AIC (BIC). Observe that a simple model of persistence (P) has been added to the set of models. It is defined by $\widehat{\mathrm{GHI}}(y, d, h+\ell)=\operatorname{GHI}(y, d, h)$. It assumes that all $\ell$-step ahead forecast values of GHI are equal to GHI at hour $h$, independent of $y$ and $d$. It is also worth mentioning that the three models "simple SP", "smart SP1", and "smart SP2" are well-specified at both sites, i.e. their specification has benefited from long work experience.

Initially, the AOD and $w$ parameters are updated each month from the aeronet database (https:aeronet.gsfc.nasa.gov/) where AOD values fluctuate between 0.1 and 0.5 and $w$ between $0.2 \mathrm{~cm}$ and $0.9 \mathrm{~cm}$. If we select constant and ill-chosen values; $\mathrm{OOD}=0.9$ and $w=0.9$, the 
Table 3: $\operatorname{nRMSE}(\ell)$ values $(\ell=1, \ldots, 6)$. MLP results are based on the best of 10 training sets with random initialization. Bold values show the best results in each row.

\begin{tabular}{|c|c|c|c|c|c|c|c|c|}
\hline \multirow{2}{*}{$\begin{array}{c}\text { Horizon } \\
(\ell)\end{array}$} & \multirow{2}{*}{$\begin{array}{c}\text { Climatology } \\
\text { TMY }\end{array}$} & \multicolumn{3}{|c|}{ Persistence } & \multirow[t]{2}{*}{$\mathrm{AR}$} & \multirow[t]{2}{*}{ MLP } & \multicolumn{2}{|c|}{ PAR } \\
\hline & & Simple P & Smart SP1 & Smart SP2 & & & $\mathrm{AIC}$ & BIC \\
\hline \multicolumn{9}{|c|}{ Ajaccio } \\
\hline 1 & 0.3554 & 0.3409 & 0.1978 & 0.1950 & 0.1965 & 0.1961 & 0.1908 & 0.1838 \\
\hline 2 & 0.3354 & 0.5938 & 0.3120 & 0.2683 & 0.2642 & 0.2661 & 0.2640 & 0.2639 \\
\hline 3 & 0.3554 & 0.7974 & 0.4299 & 0.3062 & 0.3037 & 0.3027 & 0.2982 & 0.3008 \\
\hline 4 & 0.3554 & 0.9563 & 0.5666 & 0.3313 & 0.3307 & 0.3267 & 0.3160 & 0.3149 \\
\hline 5 & 0.3554 & 1.0699 & 0.7054 & 0.3486 & 0.3497 & 0.3419 & 0.3259 & 0.3282 \\
\hline 6 & 0.3554 & 1.1449 & 0.8346 & 0.3585 & 0.3627 & 0.3540 & 0.3353 & 0.3383 \\
\hline \multicolumn{9}{|c|}{ Bastia } \\
\hline 1 & 0.3606 & 0.3609 & 0.2435 & 0.2103 & 0.2231 & 0.2235 & 0.1968 & 0.1972 \\
\hline 2 & 0.3606 & 0.6184 & 0.4013 & 0.2942 & 0.2931 & 0.2909 & 0.2660 & 0.2678 \\
\hline 3 & 0.3606 & 0.8261 & 0.5545 & 0.3384 & 0.3298 & 0.3269 & 0.2995 & 0.3048 \\
\hline 4 & 0.3606 & 0.9853 & 0.7095 & 0.3629 & 0.3515 & 0.3456 & 0.3205 & 0.3271 \\
\hline 5 & 0.3606 & 1.0963 & 0.8540 & 0.3745 & 0.3642 & 0.3593 & 0.3331 & 0.3425 \\
\hline 6 & 0.3606 & 1.1676 & 0.9821 & 0.3781 & 0.3725 & 0.3677 & 0.3409 & 0.3495 \\
\hline
\end{tabular}

one-step ahead $(\ell=1)$ nRMSE increases to 0.2394 for SP1, to 0.2300 for MLP, and to 0.2481 for AR. In this case, the accuracy of the CS is poor and widely influences the forecasts. For $\ell=6$ the results are worse, i.e. 0.9927 (SP1), 0.4404 (MLP), and 0.4571 (AR). This confirms that the accuracy of the CS model plays an important role in establishing its reliability, and that a PAR model is all the more an interesting alternative.

As compared to Ajaccio, the nRMSE results are slightly worse for Bastia. The reason is that as the nebulosity becomes more important for this site, the CS model is very difficult to optimize. Moreover, the training set covers only 5 years of data. In summary, even when a CS model is well-specified, PAR models give the best forecasting results for all horizons. GM models (AR and MLP) give good nRMSE results, albeit with some variability. Overall, SP2 based on TMY gives lower nRMSE results than SP1 based on CS. The use of TMY with PAR methodology improves the forecasting results. In particular, for Ajaccio nRMSE $(1)=0.1838$ (PAR-BIC) with TMY and 0.2125 without. This effect is equivalent for the other forecast horizons $\ell$, i.e. the gain of including TMY in a PAR model specification comes close to 4 percentage points. 


\subsection{Forecasts intervals}

The reliability of a FI can be assessed by nMIL and PICP. An optimal methodology is associated with a high PICP (close to 100\%) and a low nMIL. The choice of a lower (L) and upper (U) FI limit is essential here. To explore the null hypothesis that the $\operatorname{GHI}(y, d, h)$ data comes from a symmetrical distribution (skewness) with an unknown median, we used the triplestest available from MATLAB Central. At the 5\% nominal significance level, the test statistic indicates that the null hypothesis cannot be rejected for $h=6,7,8,17$ and 20. For the remaining 12 series, we obtained small $p$-values casting doubt about the validity of the null hypothesis. Nevertheless, we set $\mathrm{L}_{t+\ell}(h) \equiv-\mathrm{U}_{t+\ell}(h)$ throughout the rest of the analysis.

Figure 1 shows a plot of $\operatorname{nMIL}(\ell)$ versus $\operatorname{PICP}(\ell)(\ell=1, \ldots, 6)$ for Ajaccio aggregated over all values of $h \in[4,20]$. Clearly, the best compromise between these accuracy measures is for $1 \leq \mathrm{U}_{t+\ell}(h) \leq 2$ with PICP in the range $[0.7,0.8]$ and nMIL $\in[0.3,0.6]$ for $\mathrm{U}_{t+\ell}(h)=1$, while $\mathrm{PICP} \in[0.8,0.9]$ and $\mathrm{nMIL} \in[0.7,1.2]$ for $\mathrm{U}_{t+\ell}(h)=2$. Interestingly, mixed results emerge from using BFIs. For instance, at $\alpha=0.05$ and taken across all values of $h, \operatorname{nMIL}(1)=0.02$ and $\operatorname{PICP}(1)=0.46$ with the mean length of the $\mathrm{BFI}_{0.05}(1)$ interval ranging between $1.97(h=4)$ and $302.9(h=13)$.

The bootstrapped nMIL and PICP values may be tentatively compared with those obtained when $\mathrm{U}_{t+1}(h)=1.96$ which corresponds to a $95 \% \mathrm{FI}$ if, for the moment, the forecast errors are assumed to be normally distributed. Then $\mathrm{nMIL}(1)=1.1, \mathrm{PICP}(1)=0.92$ and the mean length of the FI, averaged over all $h$, equals 84.9. So, with bootstrapping the value of nMIL in this case is considerably smaller than in the non-bootstrapping case, but with bootstrapping there is also a marked decrease in the value of PICP. This observation applies to all values of $\ell$.

Observe that the above results are taken over all values of $h$ jointly. Since the selected PAR orders are different for each $h$, it is interesting to present nMIL and PICP values for each $h$ separately. Table 4 shows these results for $\mathrm{U}_{t+1}(h)=1,1.5$, and 2 . We see that for $h=8, \ldots, 16$ the one-step ahead PICP values for Ajaccio and Bastia are very close to $100 \%$. The nMIL values on the other hand increase for $h=8, \ldots, 16$ with values for Bastia about two times larger than those for Ajaccio. At this point it is worth noting that the choice of the upper and lower FI bands depends on the operational system in place, and on the prerogative of the network manager. But the results in Figure 1 and Table 4 show that PAR models can result in quite accurate one-step ahead forecasts with high confidence.

Table 5 shows the impact of TMY on the PAR forecasts. Introducing TMY in a PAR model 


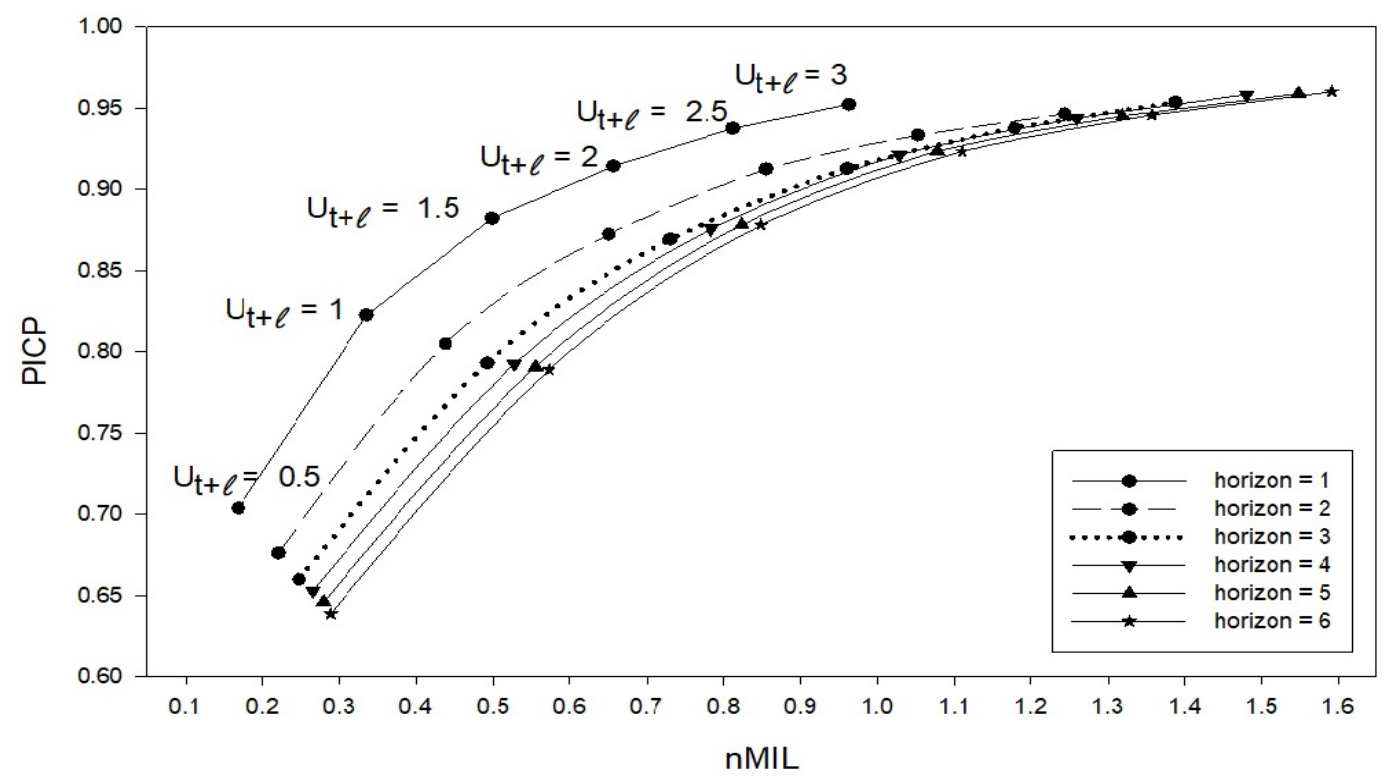

Figure 1: $\operatorname{nMIL}(\ell)$ versus $\operatorname{PICP}(\ell)$ for various values of $\mathrm{U}_{t+\ell}(h)(\ell=1, \ldots, 6)$ for Ajaccio. PAR orders are selected by BIC.

Table 4: nMIL and PICP values (in percentages) for different values of $\mathrm{U}_{t+1}(h)(h=4, \ldots, 20)$.

\begin{tabular}{|c|c|c|c|c|c|c|c|c|c|c|c|c|}
\hline \multirow[b]{3}{*}{$h$} & \multicolumn{6}{|c|}{ Ajaccio } & \multicolumn{6}{|c|}{ Bastia } \\
\hline & \multirow{2}{*}{\multicolumn{2}{|c|}{$\frac{\mathrm{U}_{t+1}(h)=1}{\mathrm{nMIL} \text { PICP }}$}} & \multicolumn{2}{|c|}{$\mathrm{U}_{t+1}(h)=1.5$} & \multicolumn{2}{|c|}{$\mathrm{U}_{t+1}(h)=2.0$} & \multirow{2}{*}{\multicolumn{2}{|c|}{$\frac{\mathrm{U}_{t+1}(h)=1}{\mathrm{nMIL} \text { PICP }}$}} & \multicolumn{2}{|c|}{$\mathrm{U}_{t+1}(h)=1.5$} & \multicolumn{2}{|c|}{$\mathrm{U}_{t+1}(h)=2.0$} \\
\hline & & & nMIL & PICP & MIL & PICP & & & nMIL & PICP & nMIL & PICP \\
\hline 4 & 0.0 & 1.1 & 0.0 & 1.1 & 0.0 & 1.1 & 0.4 & 0.0 & 0.6 & 0.0 & 0.9 & 0.0 \\
\hline 5 & 0.7 & 37.5 & 1.1 & 37.5 & 1.5 & 37.5 & 1.4 & 24.1 & 2.1 & 24.1 & 2.7 & 24.1 \\
\hline 6 & 6.6 & 62.5 & 9.7 & 62.5 & 12.8 & 62.5 & 12.8 & 55.9 & 18.8 & 55.9 & 24.6 & 55.9 \\
\hline 7 & 16.4 & 78.6 & 24.2 & 78.6 & 31. & 78.6 & 32.5 & 78.4 & 48.0 & 78.4 & 63.2 & 78.4 \\
\hline 8 & 27.7 & 98.4 & 40.7 & 98 & 5 & 98.4 & 58.9 & 97.5 & 86.6 & 7.5 & 113.2 & 97.5 \\
\hline 9 & 40.8 & 98.9 & 60.5 & 98.9 & 79.9 & 98.9 & 84.0 & 99.5 & 124.4 & 99.5 & 163.9 & 99.5 \\
\hline 10 & 49.3 & 99.2 & 73.5 & 99.2 & 97.4 & 99.2 & 105.1 & 100.0 & 156.5 & 100.0 & 207.0 & 100.0 \\
\hline 11 & 58.3 & 99.7 & 86.9 & 99.7 & 115.1 & 99.7 & 132.7 & 99.7 & 197.6 & 99.7 & 261.4 & 99.7 \\
\hline 12 & 63.3 & 100.0 & 94.2 & 100.0 & 124.6 & 100.0 & 132.2 & 99.7 & 196.7 & 99.7 & 260.1 & 99.7 \\
\hline 13 & 64.2 & 100.0 & .3 & 100.0 & 125.5 & 100.0 & 136.9 & 100.0 & 203.4 & 100.0 & 268.6 & 100.0 \\
\hline 14 & 60.1 & 100.0 & 89.0 & 100.0 & $11^{\prime}$ & 100.0 & 128.0 & 99.7 & 188.9 & 99,7 & 247 & 99.7 \\
\hline 15 & 53.1 & 100.0 & 78.6 & 100.0 & 103.4 & 100.0 & 111.5 & 99.2 & 164.1 & 99.2 & 214.6 & 99.2 \\
\hline 16 & 38.8 & 97.3 & 57.0 & 97.3 & 74.7 & 97.3 & 75.6 & 95.6 & 11.2 & 95.6 & 145.5 & 95.6 \\
\hline 17 & 24.6 & 83.6 & 35.8 & 83.6 & 46.7 & 83.6 & 47.4 & 80.5 & 69.6 & 80.5 & 91.1 & 80.5 \\
\hline 18 & 12.6 & 58.4 & 18.5 & 58.4 & 24.3 & 58.4 & 23.3 & 59.2 & 34.5 & 59.2 & 45.5 & 59.2 \\
\hline 19 & 4. & 34.0 & 6. & 34.0 & 8. & 34.0 & 8.1 & 44.4 & $12 .($ & 44.4 & 15.9 & 44.4 \\
\hline 20 & 0.5 & 0.0 & 0.8 & 0.0 & 1.1 & 0.0 & 1.2 & 21.4 & 1.8 & 21.4 & 2.4 & 21.4 \\
\hline
\end{tabular}


Table 5: Forecast interval evaluation measures for the fitted PAR models; $\mathrm{U}_{t+\ell}(h)=2$.

\begin{tabular}{|c|c|c|c|c|c|c|}
\hline \multirow{3}{*}{$\begin{array}{c}\text { Horizon } \\
(\ell)\end{array}$} & \multicolumn{2}{|c|}{$\operatorname{PICP}(\%)$} & \multirow[t]{3}{*}{ Gain(\%) } & \multicolumn{2}{|c|}{ nMIL(\%) } & \multirow[t]{3}{*}{ Gain(\%) } \\
\hline & Without & With & & Without & With & \\
\hline & TMY & TMY & & TMY & TMY & \\
\hline & \multicolumn{6}{|c|}{ Ajaccio } \\
\hline 1 & 91.0 & 91.4 & +0.4 & 70.3 & 65.7 & -4.6 \\
\hline 2 & 89.3 & 91.3 & +2.0 & 92.5 & 85.6 & -6.9 \\
\hline 3 & 88.2 & 91.2 & +3.0 & 104.5 & 96.0 & -8.5 \\
\hline 4 & 87.2 & 92.1 & +4.9 & 112.6 & 102.8 & -9.8 \\
\hline 5 & 86.8 & 92.4 & +5.6 & 118.4 & 107.8 & -10.6 \\
\hline \multirow[t]{2}{*}{6} & 86.6 & 92.3 & +5.7 & 122.5 & 111.0 & -11.5 \\
\hline & \multicolumn{6}{|c|}{ Bastia } \\
\hline 1 & 95.0 & 94.7 & -0.3 & 78.3 & 70.4 & -7.9 \\
\hline 2 & 94.3 & 94.3 & 0 & 102.3 & 91.6 & -10.7 \\
\hline 3 & 94.1 & 94.1 & 0 & 114.6 & 102.4 & -12.2 \\
\hline 4 & 94.0 & 94.0 & 0 & 122.3 & 109.3 & -13.0 \\
\hline 5 & 94.0 & 94.1 & +0.1 & 127.6 & 113.8 & -13.8 \\
\hline 6 & 94.2 & 94.2 & 0 & 131.0 & 116.8 & -14.2 \\
\hline
\end{tabular}

specification, reduces the values of $\operatorname{nMIL}(\ell)$ significantly; between $4.6 \%$ and $11.5 \%$ for Ajaccio, and between $7.9 \%$ and $14.2 \%$ for Bastia. Interestingly, for Bastia the $\operatorname{PICP}(\ell)$ values remain constant and close to $95 \%$ across all values of $\ell$. This phenomenon is quite different for Ajaccio with decreasing values of $n M I L(\ell)$ and increasing values of $\operatorname{PICP}(\ell)$.

\section{Concluding Remarks}

We have introduced the PAR family of models and applied these models to forecast GHI time series. PAR models are intended for periodic time series. They are not restricted to stationary time series. In contrast, classical models like AR, and MLP need a preprocessing transformation to make the GHI series stationary. Thereby, in the PAR case, the use of a knowledge-based model is not necessary. Usually a CS radiation model is fitted to the data to remove certain components, and hence the initial time series is transformed in a new stationary series of CSIs.

This transformation is efficient if all parameters are perfectly known. But if this is not the case, the introduction of a CS model can become ineffective and can even give rise to an additional source of error. The results obtained in this study show that even if a CS model is well-specified, AR and MLP coupled with CS are less efficient than a PAR model. Indeed, for all the horizons, 
PAR gives the best results even if the improvement is small compared with other models. If a CS model is not perfectly known for a site, a PAR model becomes an interesting alternative with minimal forecast errors. The model allows FIs and through the use of upper and lower forecast bands the length of the FIs can be adjusted to gain forecast efficiency.

A related study is by Pedro et al. (2018) based on 5 minutes GHI and direct normal irradiance (DNI) data obtained from Folsom, CA, USA. With the purpose of building an intra-hour forecasting model, they use two machine learning algorithms, i.e. k-nearest-neighbors $(\mathrm{kNN})$ and gradient boosting. At a nominal coverage of $80 \%$ and a 30 minute forecast horizon, the best result is obtained for a kNN method with cloud cover information derived from sky images (Pedro et al., 2018, Table 6). The reported values are PICP $=81.5 \%$ and nMIL $=9.9 \%$, with nMIL called PINAW (Prediction Interval Normalized) by these authors. In contrast, a PAR model gives 84.7\% (PICP) and 73.5\% (nMIL) at a one hour forecast horizon while with bootstrapping we have $49.5 \%$ (PICP) and 3.9\% (nMIL). It is evident that these results should be interpreted with care. Especially, since data location, data frequency, forecast horizon, and forecasting methods are different. Nevertheless, it is clear that PAR models perform pretty well in this special case. Moreover, PAR models need no additional forecast information in the form of sky images.

In summary, while PAR models are known since many years, they are rarely used for GHI forecasting. A PAR model is a good and recommended model when a forecasting tool must be developed for a new meteorological site. In particular, if there is not sufficient hindsight and historical data for which a CS model is not well-defined by, for instance, lack of meteorological data. This frequently happens when a new project of photovoltaic plant implementation occurs at a new site. Some further research is needed for greater validation of the proposed methodology. For instance, comparing PAR forecasts with other models/methods, using GHI data obtained from a larger set of meteorological sites, and studying different time granularities 


\section{References}

David, M., Ramahatana, F., Trombe, P.-J., Lauret, P., 2016. Probabilistic forecasting of the solar irradiance with recursive ARMA and GARCH models. Sol. Energy, 133, 55-72. DOI: $10.1016 / j$. solener.2016.03.064.

Diagne, M., David, M., Lauret, P., Boland, J., Schmutz, N., 2013. Review of solar irradiance forecasting methods and a proposition for small-scale insular grids. Renew. Sustain. Energy Rev., 27, 65-76. DOI: 10.1016/j.rser.2013.06.042.

De Gooijer, J.G., 2017. Elements of Nonlinear Time Series Analysis and Forecasting. Springer Series in Statistics, Springer-Verlag, New York. DOI: 10.1007/978-3-319-43252-6.

Franses, P.H., Paap, R., 1994. Model selection in periodic autoregressions. Oxf. Bull. Econ. Stat. 56(4), 421-439. DOI: 10.1111/j.1468-0084.1994.tb00018.x.

Gelaro, R., McCarty, W., Suárez, M.J., Todling, R., Molod, A., Takacs, L., Randles, C.A., Darmenov, A., Bosilovich, M.G., Reichle, R., Wargan, K., Coy, L., Cullather, R., Draper, C., Akella, S., Buchard, V., Conaty, A., da Silva, A.M., Gu, W., Kim, G.-K., Koster, R., Lucchesi, R., Merkova, D., Nielsen, J.E., Partyka, G., Pawson, S., Putman, W., Rienecker, M., Schubert, S.D., Sienkiewicz, M., Zhao, B., 2017. The modern-ear retrospective analysis for research and applications, Version 2 (MERRA-2). J. Climate, 30, 5419-5454. DOI: 10.1175/ JCLI-D-16-0758.1.

Gladhyšhev, E.G., 1961. Periodically correlated random sequences. Sov. Math. Doklady, 2, 385388.

Grantham, A.P., Gel, Y.R., Boland, J.W., 2016. Nonparametric short-term probabilistic forecasting for solar radiation, Sol. Energy, 133, 465-475. DOI: 10.1016/j . solener.2016.04.011.

Grantham, A.P., Pudney, P.J., Boland, J.W., 2018. Generating synthetic sequences of global horizontal irradiation. Sol. Energy, 162, 500-509. DOI: 10.1016/j.solener.2018.01.044.

Hokoi, S., Matsumoto, M., Ihara, T., 1990. Statistical time series models of solar radiation and outdoor temperature - Identification of seasonal models by Kalman filter. Energy Build., 15(3-4), 373-383. DOI: 10.1016/0378-7788(90)90011-7.

Ineichen, P., 2006. Comparison of eight clear sky broadband models against 16 independent data banks. Sol. Energy, 80(4), 468-478. DOI: 10.1016/j. solener.2005.04.018. 
Ineichen, P., 2008. A broadband simplified version of the solis clear sky model. Sol. Energy, 82(8), 758-762. DOI: 10.1016/j.solener.2008.02.009.

Ineichen, P., Guisan, O., Perez, R., 1990. Ground-reflected radiation and albedo. Sol. Energy, 44(4), 207-214. DOI: 10.1016/0038-092x(90)90149-7.

Korany, M., Boraiy, M., Eissa, Y., Aoun, Y., Abdel Wahab, M.M., Alfaro, S.C., Blanc, P., ElMetwally, M., Ghedira, H., Hungershoefer, K., and others, 2016. A database of multi-year (2004-2010) quality-assured surface solar hourly irradiation measurements for the Egyptian territory. Earth Syst. Sci. Data, 8(1), 105-113. DOI: 10.5194/essdd-8-737-2015.

Lauret, P., Voyant, C., Soubdhan, T., David, M., Poggi, P., 2015. A benchmarking of machine learning techniques for solar radiation forecasting in an insular context. Sol. Energy, 112, 446-457. DOI: 10.1016/j.solener.2014.12.014.

McLeod, A.I., 1994. Diagnostic checking of periodic autoregression models with applications. J. Time Ser. Anal., 15(2), 221-233. DOI: 10.1111/j.1467-9892.1994.tb00186.x.

Mueller, R.W., Dagestad, K.F., Ineichen, P., Schroedter-Homscheidt, M., Cros, S., Dumortier, D., Kuhlemann, R., Olseth, J.A., Piernavieja, G., Reise, C., Wald, L., Heinemann, D., 2004. Rethinking satellite-based solar irradiance modelling: The SOLIS clear-sky module. Remote Sens. Environ., 91(2), 160-174. DOI: 10.1016/s0034-4257(04)00069-0.

Notton, G., Poggi, P., Cristofari, C., 2006. Predicting hourly solar irradiations on inclined surfaces based on the horizontal measurements: Performances of the association of wellknown mathematical models. Energy Convers. Manag., 47(13-14), 1816-1829. DOI: 10.1016/ j.enconman.2005.10.009.

Notton, G., Nivet, M.-L., Voyant, C., Paoli, C., Darras, C., Motte, F., Fouilloy, A., 2018. Intermittent and stochastic character of renewable energy sources: Consequences, cost of intermittence and benefit of forecasting. Renew. Sustain. Energy Rev., 87, 96-105. DOI: $10.1016 / j . r s e r .2018 .02 .007$.

Notton, G., Voyant, C., 2018. Forecasting of intermittent solar energy resource (Chapter 3). In I. Yahyaoui (Ed.), Advances in Renewable Energies and Power Technologies, Volume 1: Solar and Wind Energies, Elsevier, pp. 77-114. DOI: 10.1016/b978-0-12-812959-3.00003-4.

Pan, L., Politis, N., 2016. Bootstrap prediction intervals for linear, nonlinear and nonparametric autoregressions. J. Statist. Plann. Inference, 177, 1-27. DOI: 10.1016/j · jspi.2014.10.003. 
Pedro, H.T.C., Coimbra, C.F.M., David, M., Lauret, P., 2018. Assessment of machine learning techniques for deterministic and probabilistic intra-hour solar forecasts. Renew. Energy, 123, 191-203. DOI: 10.1016/j.renene.2018.02.006.

Ruiz-Arias, J.A., Gueymard, C.A., Cebecauer, T., 2017. Worldwide multi-model intercomparison of clear-sky solar irradiance predictions. AIP Conference Proceedings 1850, 140018. DOI: 10. $1063 / 1.4984526$.

Sakai, H., 1982. Circular lattice filtering using Pagano's method. IEEE Trans. Accoustics, Speech, and Signal Processing, 30(2), 279-287. DOI: 10.1109/tassp.1982.1163874.

Shamshirband, S., Mohammadi, K., Yee, P.L., Petković, D., Mostafaeipour, A., 2015. A comparative evaluation for identifying the suitability of extreme learning machine to predict horizontal global solar radiation. Renew. Sustain. Energy Rev., 52, 1031-1042. DOI: $10.1016 / j . r s e r .2015 .07 .173$.

Tiao, G.C., Grupe, M.R., 1980. Hidden periodic autoregressive moving average models in time series data. Biometrika, 67(2), 365-373. DOI: 10.1093/biomet/67.2.365.

Ula, T.A., Smadi, A.A., 1997. Periodic stationarity conditions for periodic autoregressive moving average processes as eigenvalue problems. Water Resour. Res., 33(8), 1929-1934. DOI: 10. $1029 / 97 \mathrm{wr} 01002$.

Ursu, E., Turkman, K.F., 2012. Periodic autoregressive model identification using genetic algorithms. J. Time Ser. Anal., 33, 398-405. DOI: 10.1111/j.1467-9892.2011.00772.x.

Voyant, C., Muselli, M., Paoli, C., Nivet, M.-L., 2011. Optimization of an artificial neural network dedicated to the multivariate forecasting of daily global radiation. Energy, 36(1), 348-359. DOI: $10.1016 / j \cdot$ energy $\cdot 2010 \cdot 10 \cdot 032$.

Voyant, C., Haurant, P., Muselli, M., Paoli, C., Nivet, M.-L., 2014. Time series modeling and large scale global solar radiation forecasting from geostationary satellites data. Sol. Energy, 102, 131-142. DOI: 10.1016/j.solener.2010.08.011.

Voyant, C., Soubdhan, T., Lauret, P., David, M., Muselli, M., 2015. Statistical parameters as a means to a priori assess the accuracy of solar forecasting models. Energy, 90, 671-679. DOI: $10.1016 / j$.energy . 2015.07.089. 
470 Voyant, C., Notton, G., Kalogirou, S., Nivet, M.-L., Paoli, C., Motte, F., Fouilloy, A., 2017. 471 Machine learning methods for solar radiation forecasting: A review. Renew. Energy, 105, 569$472 \quad 582$. DOI: $10.1016 / j . r e n e n e .2016 .12 .095$. 